\title{
Review Article \\ Controllability Problem of Fractional Neutral Systems: A Survey
}

\author{
Artur Babiarz and Michał Niezabitowski \\ Institute of Automatic Control, Silesian University of Technology, 16 Akademicka St., 44-100 Gliwice, Poland \\ Correspondence should be addressed to Michał Niezabitowski; michal.niezabitowski@polsl.pl \\ Received 4 August 2016; Revised 25 October 2016; Accepted 30 October 2016; Published 18 January 2017 \\ Academic Editor: Leonid Shaikhet
}

Copyright (c) 2017 Artur Babiarz and Michał Niezabitowski. This is an open access article distributed under the Creative Commons Attribution License, which permits unrestricted use, distribution, and reproduction in any medium, provided the original work is properly cited.

\begin{abstract}
The following article presents recent results of controllability problem of dynamical systems in infinite-dimensional space. Generally speaking, we describe selected controllability problems of fractional order systems, including approximate controllability of fractional impulsive partial neutral integrodifferential inclusions with infinite delay in Hilbert spaces, controllability of nonlinear neutral fractional impulsive differential inclusions in Banach space, controllability for a class of fractional neutral integrodifferential equations with unbounded delay, controllability of neutral fractional functional equations with impulses and infinite delay, and controllability for a class of fractional order neutral evolution control systems.
\end{abstract}

\section{Introduction}

Controllability plays a very important role in various areas of engineering and science. In particular in control systems many fundamental problems of control theory, such as optimal control, stabilizability, or pole placement can be solved with assumption that the system is controllable $[1,2]$. Controllability in general means that there exists a control function which steers the solution of the system from its initial state to a final state using a set of admissible controls, where the initial and final states may vary over the entire space. A standard approach is to transform the controllability problem into a fixed point problem for an appropriate operator in a functional space. There are many papers devoted to the controllability problem, in which authors used the theory of fractional calculus [3-13] and a fixed point approach [14-23].

The subject of fractional calculus and its applications has gained a lot of importance during the past four decades. This was mainly because it has become a powerful tool in modeling several complex phenomena in numerous seemingly diverse and widespread fields such as engineering, chemistry, mechanics, aerodynamics, and physics [24-32].

For infinite-dimensional systems two basic concepts of controllability can be distinguished: approximate and exact controllability, as in infinite-dimensional spaces there exist linear subspaces which are not closed. Approximate controllability enables steering the system to an arbitrarily small neighbourhood of final state. The second one, that is, exact controllability, means that system can be steered to arbitrary final state. From these definitions it is obvious that approximate controllability is essentially weaker notion than exact controllability. In the case of finite-dimensional systems notions of approximate and exact controllability coincide.

Many control systems arising from realistic models can be described as partial fractional differential or integrodifferential inclusions [33-36]. In [37] authors present a new approach to obtain the existence of mild solutions and controllability results. For this purpose they avoid hypotheses of compactness on the semigroup generated by the linear part and any conditions on the multivalued nonlinearity expressed in terms of measures of noncompactness. Author of [38] focuses on fractional evolution equations and inclusions. Moreover author presents their applications to control theory. The existence of solutions for fractional semilinear differential or integrodifferential equations has been studied by many authors [39-43].

The impulsive differential systems can be used to model processes which are subject to sudden changes and which cannot be described by classical differential systems [44]. The controllability problem for impulsive differential and integrodifferential systems in Banach spaces has been discussed 
in [45]. Papers [46, 47] are devoted to the controllability of fractional evolution systems. The problem of controllability and optimal controls for functional differential systems has been extensively studied in many papers [48-50].

1.1. Motivation. Controllability is one of the properties of dynamical systems that is continuously studied by control theory scientists. In case of infinite-dimensional systems there are many articles tackling this problem, in particular for approximate controllability, exact controllability, and relative controllability. This field can be divided based on the nature of controllability, but also on the basis of main equations describing a system of interest as well as the space in which the mathematical model is described. Additionally researchers frequently use different fixed point theorems for finding controllability conditions. That introduces high intricacy of problems which one can encounter during an analysis of a particular problem. The main purpose of this work is to perform a survey on the main types of equations describing dynamical systems based on a definition of a fractional order derivative. Additionally, as a result this work performs a systematization of knowledge in the field of controllability fractional systems, which by itself becomes a major discipline in the realm of control theory. This work shows schematics present in the analysis of controllability problems as well as points out which fixed point theorems are particularly useful.

\section{Basic Notations}

Let us introduce the following necessary notations.

(i) $(X,\|\cdot\|)$ is a Banach space.

(ii) $(H,\|\cdot\|)$ is a Hilbert space.

(iii) $J$ is a bounded and closed interval.

(iv) $x: J \rightarrow H$ is a measurable function and Bochner integrable [51].

(v) $C(J, H)$ is the Hilbert space of all continuous functions from $J$ into $H$ with the norm $\|x\|_{\infty}=$ $\sup \{\|x(t)\|: t \in J\}$.

(vi) $L(H)$ denotes the Hilbert space of bounded linear operators from $H$ to $H$.

(vii) $U$ is a Hilbert space.

(viii) $L^{1}(J, H)$ denotes the Hilbert space of measurable functions $x: J \rightarrow H$ which are Bochner integrable normed by $\|x\|_{L^{1}}=\int_{J}\|x(t)\| d t$ for all $x \in L^{1}(J, H)$.

(ix) $L^{2}(J, U)$ is a space of all strongly measurable functions $u: J \rightarrow U$.

(x) $B_{r}(x, H)$ is the closed ball with centre at $x$ and radius $r>0$ in $H$.

(xi) $\mathscr{P}(H)$ denotes the class of all nonempty subsets of $H$.

(xii) $\mathscr{P}_{b d, c l}(H), \quad \mathscr{P}_{c p, c v}(H), \mathscr{P}_{b d, c l, c v}(H)$, and $\mathscr{P}_{c d}(H)$ denote, respectively, the families of all nonempty bounded-closed, compact-convex, bounded-closedconvex, and compact-acyclic [52] subsets of $H$.

(xiii) $F$ is completely continuous. (xiv) $G: J \rightarrow \mathscr{P}_{b d, c l, c v}(H)$ is measurable multivalued map.

(xv) $t \mapsto D(x, G(t))$ is a measurable function on $J$.

(xvi) $B$ is a bounded linear operator from $U$ to $H$.

(xvii) $M_{B}=|B|$.

(xviii) If $T$ is a uniformly bounded and analytic semigroup with infinitesimal generator $A$ such that $0 \in \rho(A)$ then it is possible to define the fractional power $(-A)^{\alpha}$, for $0<\alpha \leqslant 1$, as a closed linear operator on its domain $D\left((-A)^{\alpha}\right)$. Furthermore, the subspace $D\left((-A)^{\alpha}\right)$ is dense in $X$ and the expression

$$
\|x\|_{\alpha}:=\left\|(-A)^{\alpha} x\right\|, \quad x \in D\left((-A)^{\alpha}\right)
$$

defines a norm on $D\left((-A)^{\alpha}\right)$. Hereafter we represent by $X_{\alpha}$ the space $D\left((-A)^{\alpha}\right)$ endowed with the norm $\|\cdot\|_{\alpha}$.

(xix) $M$ is constant number such that $|T(t)| \leqslant M$.

(xx) ${ }^{c} D_{t}^{\alpha} \xi(t)=\int_{0}^{t} g_{n-\alpha}(t-s)$ represents the Caputo derivative of order $\alpha>0$ defined by

$$
{ }^{c} D_{t}^{\alpha} \xi(t)=\int_{0}^{t} g_{n-\alpha}(t-s) \frac{d^{n}}{d s^{n}} \xi(t-s) d s,
$$

where $n$ is the smallest integer greater than or equal to $\alpha, \Gamma(\cdot)$ is the gamma function, and $g_{\beta}(t):=$ $t^{\beta-1} / \Gamma(\beta), t>0, \beta \geq 0$.

(xxi) $\mathscr{R}_{\alpha}(t)$ and $S_{\alpha}(t)$ are the operator families defined by

$$
\begin{aligned}
\mathscr{R}_{\alpha}(t) & := \begin{cases}\frac{1}{2 \pi i} \int_{\Gamma_{r, \theta}} e^{\lambda t} G_{\alpha}(\lambda) d \lambda & \text { for } t>0, \\
I & \text { for } t=0,\end{cases} \\
S_{\alpha}(t) x & :=\int_{0}^{t} g_{\alpha-1}(t-s) \mathscr{R}_{\alpha}(s) d s
\end{aligned}
$$

for $\alpha \in(1,2)$ and each $t \geq 0$.

(xxii) $0<t_{1}<\cdots<t_{m}<b$ are fixed points.

(xxiii) $x\left(t_{k}^{-}\right)$and $x\left(t_{k}^{+}\right)$represent the right and left limits of $x(t)$ at $t=t_{k}$, respectively.

(xxiv) $\Gamma_{\tau}^{b}, \Gamma_{t_{k-1}}^{t_{k}}$, and $R\left(a, \Gamma_{t_{k-1}}^{t_{k}}\right)$ are the operators defined by

$$
\Gamma_{\tau}^{b}=\int_{\tau}^{b} S_{\alpha}(b-s) B B^{*} S_{\alpha}^{*}(b-s) d s,
$$

$$
0 \leq \tau \leq b,
$$

$$
\Gamma_{t_{k-1}}^{t_{k}}=\int_{t_{k-1}}^{t_{k}} S_{\alpha}\left(t_{k}-s\right) B B^{*} S_{\alpha}^{*}\left(t_{k}-s\right) d s,
$$

$$
k=1,2, \ldots, m, m+1,
$$

$$
\begin{aligned}
& R\left(a, \Gamma_{t_{k-1}}^{t_{k}}\right)=\left(a I+\Gamma_{t_{k-1}}^{t_{k}}\right)^{-1} \\
& \qquad \text { for } a>0, k=1,2, \ldots, m, m+1,
\end{aligned}
$$

where $B^{*}$ denotes the adjoint of $B$.

Below we present definition of phase space. 
Definition 1 (see [53]). Suppose that $h:(-\infty, 0] \rightarrow(0, \infty)$ is a continuous function with $l=\int_{-\infty}^{0} h(t) d t<\infty$. For all $a>0$, one defines

$$
\begin{gathered}
\mathscr{B}=\{\psi:[-a, 0] \longrightarrow X \text { such that } \psi(t) \\
\text { is bounded and measurable }\}
\end{gathered}
$$

and equips the space $\mathscr{B}$ with the norm $\|\psi\|_{[-a, 0]}=$ $\sup _{s \in[-a, 0]}\|\psi(s)\|, \forall \psi \in \mathscr{B}$. Let us define the phase space

$$
\begin{aligned}
& \mathscr{B}_{h}=\{\psi:(-\infty, 0] \longrightarrow X \text { such that, for any } c \\
&\left.>0,\left.\quad \psi\right|_{[-c, 0]} \in \mathscr{B}, \int_{-\infty}^{0} h(s)\|\psi\|_{[s, 0]} d s<\infty\right\} .
\end{aligned}
$$

If $\mathscr{B}_{h}$ is endowed with the norm $\|\psi\|_{\mathscr{B}_{h}}=$ $\int_{-\infty}^{0} h(s)\|\psi\|_{[s, 0]} d s, \forall \psi \in \mathscr{B}$, then it is clear that $\left(\mathscr{B}_{h},\|\cdot\|_{\mathscr{B}_{h}}\right)$ is a Banach space.

Now we consider the space

$$
\begin{aligned}
\mathscr{B}_{b} & =\left\{\psi:(-\infty, b] \longrightarrow X \text { such that } x_{k}\right. \\
& \in C\left(J_{k}, X\right) \text { and there exist } x\left(t_{k}^{+}\right), x\left(t_{k}^{-}\right) \text {with } x\left(t_{k}\right) \\
& \left.=x\left(t_{k}^{-}\right), x_{0}=\phi \in \mathscr{B}_{h}, k=0,1, \ldots, m\right\},
\end{aligned}
$$

where $x_{k}$ is the restriction of $x$ to $J_{k}=\left(t_{k}, t_{k+1}\right], \quad k=0,1$, $\ldots, m$. Set $|\cdot|_{b}$ be a seminorm in $\mathscr{B}_{b}$ defined by

$$
\|x\|_{b}=\left\|x_{0}\right\|_{\mathscr{B}_{h}}+\sup \{|x(s)|: s \in[0, b]\}, \quad x \in \mathscr{B}_{b} .
$$

Definition 2 (see [54]). Let $(\mathscr{X}, d)$ be a metric space and $F$ : $\mathscr{X} \rightarrow \mathscr{X}$. One will say that operator $F$ is a contraction if there exists some $k \in(0,1)$ such that

$$
\bigwedge_{x, y \in \mathscr{X}} d(F(x), F(y)) \leq k d(x, y) .
$$

Theorem 3 (Krasnoselskii's fixed point theorem). Let $\Omega$ be a bounded, closed, and convex subset of $X$. Let $F_{1}, F_{2}: \Omega \rightarrow X$ be two mappings such that $F_{1} x+F_{2} y \in \Omega$ for every pair $x, y \in \Omega$.

If $F_{1}$ is a contraction and $F_{2}$ is completely continuous, then the operator equation $F_{1} x+F_{2} x=x$ has a solution on $\Omega$. form.

Then, the Banach fixed point theorem has the following

Theorem 4 ((Banach fixed point theorem) [54]). Let $F$ be a contraction on $X$. Then, there exists a unique $x_{0} \in X$ such that

$$
F\left(x_{0}\right)=x_{0}
$$

\section{Selected Problems of Controllability of Fractional Order Systems}

In this section, we describe recent results of controllability problem of semilinear systems in infinite-dimensional spaces. The dynamical systems are expressed by different types of semilinear fractional order equations.

\subsection{Approximate Controllability of Fractional Impulsive Partial} Neutral Integrodifferential Inclusions with Infinite Delay in Hilbert Spaces. The authors of paper [55] derived a new set of sufficient conditions for the approximate controllability of fractional impulsive evolution system under the assumption that the corresponding linear system is approximately controllable. To do this they considered the approximate controllability of a class of fractional impulsive partial neutral integrodifferential inclusions with infinite delay in Hilbert spaces of the form

$$
\begin{array}{r}
{ }^{c} D^{\alpha} N\left(x_{t}\right) \in A N\left(x_{t}\right)+\int_{0}^{t} Q(t-s) N\left(x_{s}\right) d s+B u(s)+F\left(t, x_{t}, \int_{0}^{t} h\left(t, x, x_{s}\right) d s\right), \\
t \in J=[0, b], t \neq t_{k}, \Delta x\left(t_{k}\right)=I_{k}\left(x_{t_{k}}\right), k=1, \ldots, m, x_{0} \in \varphi \in \mathscr{B}_{h}, x^{\prime}(0)=0,
\end{array}
$$

where

(i) $x(\cdot)$ takes values in the Hilbert space $H$;

(ii) $\varphi$ is an initial condition;

(iii) $\alpha \in(1,2)$;

(iv) $A,(\mathrm{Q}(t))_{t \geq 0}$, are closed linear operators defined on a common domain which is dense in $(H,\|\cdot\|)$;

(v) $u \in L^{2}(J, U)$ is admissible control functions; (vi) the function $x_{t}:(-\infty, 0] \rightarrow H$ defined by $x_{t}(\theta)=$ $x(t+\theta), \theta \in(-\infty, 0]$ belongs to some abstract phase space $\mathscr{B}_{h}$;

(vii) $F: J \times \mathscr{B}_{h} \times H \rightarrow P(H)$ is a bounded, closed, convexvalued, multivalued map;

(viii) $P(H)$ is the family of all nonempty subsets of $H$;

(ix) $G: J \times \mathscr{B}_{h} \rightarrow H, N(\psi)=\psi(0)+G(t, \psi), \psi \in \mathscr{B}_{h}$, and $I_{k}: \mathscr{B}_{h} \rightarrow H(k=1, \ldots, m)$ are functions subject to some additional conditions which will be given later. 
In order to obtain theorem about existing of solutions and a new set of sufficient conditions for the approximate controllability of system (11) we recall few important definitions and present necessary conditions.

Definition 5. The set

$$
\mathscr{B}_{h}\left(b, x_{0}\right)=\left\{x_{b}\left(x_{0} ; u\right)(0): u(\cdot) \in L^{2}(J, U)\right\}
$$

is called the reachable set of system (11) at terminal time $b$. Its closure in $H$ is denoted by $\overline{\mathscr{B}_{h}\left(b, x_{0}\right)}$.

Definition 6. System (11) is said to be approximately controllable on the interval $[0, b]$ if $\overline{\mathscr{B}_{h}\left(b, x_{0}\right)}=H$.

Condition 1. The operator families $\mathscr{R}_{\alpha}(t)$ and $S_{\alpha}(t)$ are compact for all $t>0$, and there exist constants $M$ and $\delta$ such that $\left\|\mathscr{R}_{\alpha}(t)\right\|_{L(H)} \leq M e^{\delta t}$ and $\left\|S_{\alpha}(t)\right\|_{L(H)} \leq M e^{\delta t}$ for every $t \in J$.

Condition 2. The function $G: J \times \mathscr{B}_{h} \rightarrow H$ is continuous and there exists a $L>0$ such that

$$
\begin{array}{r}
\left\|G\left(t, \psi_{1}\right)-G\left(t, \psi_{2}\right)\right\| \leq L\left[\left|t_{1}-t_{2}\right|+\left\|\psi_{1}-\psi_{2}\right\|_{\mathscr{B}_{h}}\right], \\
t_{1}, t_{2} \in J, \psi_{1}, \psi_{2} \in \mathscr{B}_{h}, \\
\|G(t, \psi)\| \leq L\left(\|\psi\|_{\mathscr{B}_{h}}+1\right), \\
\quad t \in J, \psi \in \mathscr{B}_{h} .
\end{array}
$$

Condition 3. (i) For each $(t, s) \in \Lambda$ the function $h(t, s, \cdot)$ : $\mathscr{B}_{h} \rightarrow H$ is continuous and for each $x \in \mathscr{B}_{h}$, the function $h(\cdot, \cdot, x): \Lambda \rightarrow H$ is strongly measurable.

(ii) There exists a continuous function $p: \Lambda \rightarrow[0, \infty)$, such that

$$
\|h(t, s, \psi)\| \leq p(t, s) \Theta_{0}\left(\|\psi\|_{\mathscr{B}_{h}}\right)
$$

for a.e. $t, s \in J$ and $\psi \in \mathscr{B}_{h}$, where $\Theta_{0}:[0, \infty) \rightarrow(0, \infty)$ is a continuous nondecreasing function.

Condition 4. The multivalued map $F: J \times \mathscr{B}_{h} \times H \rightarrow$ $P_{b d, c l, c v}(H)$; for each $t \in J$, the function $F(t, \cdot, \cdot): \mathscr{B}_{h} \times H \rightarrow$ $P_{b d, c l, c v}(H)$ is upper semicontinuous and for each $(\psi, y) \in$ $\mathscr{B}_{h} \times H$, the function $F(\cdot, \psi, y)$ is measurable; for each fixed $(\psi, y) \in \mathscr{B}_{h} \times H$, the set

$$
\begin{aligned}
S_{F, \psi} & =\left\{f \in L^{1}(J, H): f(t)\right. \\
\in & \left.F\left(t, \psi, \int_{0}^{t} h(t, s, \psi) d s\right) \text { for a.e. } t \in J\right\}
\end{aligned}
$$

is nonempty.

Condition 5. There exists a continuous function $m: J \rightarrow$ $[0, \infty)$ and a continuous nondecreasing function $\Theta$ : $[0, \infty) \rightarrow(0, \infty)$ such that

$$
\begin{aligned}
\|F(t, \psi, y)\| & =\sup \{\|f\|: f \in F(t, \psi, y)\} \\
& \leq m(t) \Theta\left(\|\psi\|_{\mathscr{B}_{h}}+\|y\|\right),
\end{aligned}
$$

for a.e. $t \in J$ and each $\psi \in B$ and $y \in H$ with

$$
\int_{1}^{\infty} \frac{1}{s+\Theta(s)+\Theta_{0}(s)} d s=\infty .
$$

Condition 6. The functions $I_{k}: \mathscr{B}_{h} \rightarrow H$ are continuous and there exist constants $c_{k}$ such that

$$
\limsup _{\|\psi\|_{\mathscr{B}_{h}} \rightarrow \infty} \frac{\left\|I_{k}(\psi)\right\|}{\|\psi\|_{\mathscr{B}_{h}}}=c_{k}
$$

for every $\psi \in \mathscr{B}_{h}, k=1, \ldots, m$.

Lemma 7 (see [56]). Let $J$ be a compact interval and $H$ be a Hilbert space. Let $F$ be a multivalued map satisfying Condition 4 and let $P$ be a linear continuous operator from $L^{1}(J, H)$ to $C(J, H)$. Then the operator

$$
\begin{aligned}
P \circ S_{F}: C(J, H) & \longrightarrow P_{c p, c v}(H), \\
x & \longrightarrow\left(P \circ S_{F}\right)(x):=P\left(S_{F}, x\right)
\end{aligned}
$$

is a closed graph in $C(J, H) \times C(J, H)$.

Theorem 8 (see [55]). Suppose that Conditions 1-6 are satisfied and that, for all $a>0$, system (11) has at least one mild solution on $J$, provided that

$$
\max _{1 \leq k \leq m}\left\{M_{2}\left[1+K_{b}\left(M c_{k}+M L\right)\right]+M_{3} K_{b} M L\right\}<1,
$$

where $M_{2}=M N_{*}\left(1+(1 / a) M_{*}^{2} N_{*}^{2} M_{1}^{2} b\right), M_{3}=(1+(1 /$ a) $\left.M M_{*} N_{*} M_{1}^{2} b\right) N_{*}, M_{*}=M \max \left\{1, e^{\delta b}\right\}, N_{*}=\max \{1$, $\left.e^{-\delta b}\right\}$, and $M_{1}=\|B\|$.

Now we present the main result of paper [55] on the approximate controllability of system (11).

Theorem 9 (see [55]). Assume that assumptions of Theorem 8 hold and, in addition, there exists a positive constant $\widetilde{C}$ such that

$$
\begin{aligned}
\|F(t, \psi, y)\|=\sup \{\|f\|: f \in F(t, \psi, y)\} & \leq \widetilde{C}, \\
& (t, \psi, y) \in J \times \mathscr{B}_{h} \times H
\end{aligned}
$$

and the linear system corresponding to system (11) is approximately controllable on J. Then system (11) is approximately controllable on $\mathrm{J}$.

The proofs of the Theorems 8 and 9 presented in [55] are obtained with nonlinear alternative of Leray-Schauder type for multivalued maps [57].

3.2. Controllability of Nonlinear Neutral Fractional Impulsive Differential Inclusions in Banach Space. Controllability of nonlinear neutral fractional impulsive differential inclusions in Banach space was investigated in paper [53]: 


$$
\begin{aligned}
& { }^{c} D_{t}^{\alpha}\left[x(t)-g\left(t, x_{t}\right)\right] \in A x(t)+F\left(t, x_{t}\right)+(B u)(t), \\
& \quad t \in J=[0, b], t \neq t_{k}, k=1,2, \ldots, m,\left.\Delta x\right|_{t=t_{k}}=I_{k}\left(x\left(t_{k}^{-}\right)\right), k=1,2, \ldots, m, x_{0}=\phi \in \mathscr{B}_{h}, t \in J_{0}=(-\infty, 0],
\end{aligned}
$$

where

(i) $\alpha \in(0,1)$;

(ii) $x(\cdot) \in X$;

(iii) $A$ is the infinitesimal generator of an analytic semigroup of the bounded linear operator $\{T(t), t \geqslant 0\}$ in $X$;

(iv) $F: J \times \mathscr{B}_{h} \rightarrow \mathscr{P}(X)$ is a bounded, closed, convexvalued multivalued map;

(v) $g: J \times \mathscr{B}_{h} \rightarrow X$ are given functions;

(vi) $I_{k} \in C(X, X)(k=1,2, \ldots, m)$ are bound functions.

The author of [53] used the following fixed point theorem.

Theorem 10 (see [58]). Let $X$ be a Banach space. $\Phi_{1}: X \rightarrow$ $\mathscr{P}_{c l, c v, b d}(X)$ and $\Phi_{2}: X \rightarrow \mathscr{P}_{c p, c v}(X)$ are two multivalued operators satisfying the following.

(a) $\Phi_{1}$ is a contraction.

(b) $\Phi_{2}$ is completely continuous.

\section{Then either}

(i) the operator inclusion $\lambda x \in \Phi_{1} x+\Phi_{2} x$ has a solution for $\lambda=1$, or

(ii) the set $G=\left\{x \in X: \lambda x \in \Phi_{1} x+\Phi_{2} x, \lambda>1\right\}$ is unbounded.

Definition 11. A function $x:(-\infty, b] \rightarrow X$ is called a mild solution of system (22) if the following holds: $x_{0}=\phi \in \mathscr{B}_{h}$ on $(-\infty, 0],\left.\Delta x\right|_{t=t_{k}}=I_{k}\left(x\left(t_{k}^{-}\right)\right), k=1,2, \ldots, m$; the restriction of $x(\cdot)$ to the interval $[0, b)-\left\{t_{1}, t_{2}, \ldots, t_{m}\right\}$ is continuous and the integral equation

$$
\begin{aligned}
x(t)= & S_{\alpha}(t)[\phi(0)-g(0, \phi)]+g\left(t, x_{t}\right) \\
& +\int_{0}^{t}(t-s)^{\alpha-1} A T_{\alpha}(t-s) g\left(s, x_{s}\right) d s \\
& +\int_{0}^{t}(t-s)^{\alpha-1} T_{\alpha}(t-s) f(s) d s \\
& +\int_{0}^{t}(t-s)^{\alpha-1} T_{\alpha}(t-s)(B u)(s) d s \\
& +\sum_{0<t_{k}<t} S_{\alpha}\left(t-t_{k}\right) I_{k}\left(x\left(t_{k}^{-}\right)\right), \\
& t \in J, x_{0}=\phi \in \mathscr{B}_{h}, \quad t \in J_{0}
\end{aligned}
$$

is satisfied, where

$$
\begin{aligned}
& f \in S_{F, x} \\
& =\left\{f \in L^{1}(J, X): f(t) \in F\left(t, x_{t}\right), \text { for a.e. } t \in J\right\}, \\
& S_{\alpha}(t)=\int_{0}^{\infty} \xi_{\alpha}(\theta) T\left(t^{\alpha} \theta\right) d \theta, \\
& T_{\alpha}(t)=\alpha \int_{0}^{\infty} \theta \xi_{\alpha}(\theta) T\left(t^{\alpha} \theta\right) d \theta, \\
& \xi_{\alpha}(\theta)=\frac{1}{\alpha} \theta^{-1-1 / \alpha} \omega_{\alpha}\left(\theta^{-1 / \alpha}\right) \geqslant 0, \\
& \omega_{\alpha}(\theta)=\frac{1}{\pi} \sum_{n=1}^{\infty}(-1)^{n-1} \theta^{-n \alpha-1} \frac{\Gamma(n \alpha+1)}{n} \sin (n \pi \alpha), \\
& \theta \in(0, \infty),
\end{aligned}
$$

where $\xi_{\alpha}$ is probability density function defined on $(0, \infty)$; that is, $\xi_{\alpha}(\theta) \geqslant 0, \theta \in(0, \infty)$, and $\int_{0}^{\infty} \xi_{\alpha}(\theta) d \theta=1$.

The properties of the operators $S_{\alpha}(t)$ and $T_{\alpha}(t)$ can be found in [53].

In order to study the exact controllability of system (22), the following definition and conditions were made [53].

Definition 12 (see [53]). System (22) is said to be exactly controllable on the interval $J$ if for every continuous initial function, $\phi \in \mathscr{B}_{h}, x_{1} \in X$, there exists a control $u \in L^{2}(J, U)$ such that the mild solution $x(t)$ of (22) satisfies $x(b)=x_{1}$.

Condition 7. $A$ is the infinitesimal generator of an analytic semigroup of bounded linear operators $T(t)$ and $0 \in \rho(A)$; for $t \geqslant 0$, there exist constants $M$ such that $|T(t)| \leqslant M$.

Condition 8. The linear operator $W: L^{2}(J, U) \rightarrow X$ defined by

$$
W u=\int_{0}^{b}(b-s)^{\alpha-1} T(b-s) B u(s) d s
$$

has an induced inverse operator $W^{-1}$, which takes values in $L^{2}(J, U) / \operatorname{ker} W$ and there exist positive constants $M_{2}$ and $M_{3}$ such that $|B| \leqslant M_{2}$ and $\left|W^{-1}\right| \leqslant M_{3}$.

Condition 9. There exist constants $0 \leqslant \beta<1, c_{0}, c_{1}, c_{2}, L_{g}$ such that $g$ is $X_{\beta}$-valued and $(-A)^{\beta} g$ is continuous, and

(i) $\left\|(-A)^{\beta} g(t, x)\right\| \leqslant c_{1}\|x\|_{\mathscr{B}_{h}}+c_{2},(t, x) \in J \times \mathscr{B}_{h}$; 
(ii) $\left\|(-A)^{\beta} g\left(t, x_{1}\right)-(-A)^{\beta} g\left(t, x_{2}\right)\right\| \leqslant L_{g}\left\|x_{1}-x_{2}\right\|_{\mathscr{B}_{h}}$, $\left(t, x_{i}\right) \in J \times \mathscr{B}_{h}, i=1,2$, with

$$
C_{0}=L_{g} l\left[\left\|(-A)^{-\beta}\right\|+\frac{c_{1-\beta} \Gamma(1+\beta) b^{\alpha \beta}}{\beta \Gamma(1+\alpha \beta)}\right]<1 .
$$

Condition 10. There exists a constant $d_{k}$ such that $\left\|I_{k}(x)\right\| \leqslant$ $d_{k}, k=1,2, \ldots, m$ for each $x \in X$.

Condition 11. There exist an integrable function $p: J \rightarrow$ $[0, \infty)$ and a nondecreasing function $\psi: R_{+} \rightarrow(0, \infty)$ such that $\|F(t, x)\|=\sup \{|f|: f(t) \in F(t, x)\} \leqslant p(t) \psi\left(\|x\|_{\mathscr{B}_{h}}\right)$ for almost all $t \in J$ and all $x \in \mathscr{B}_{h}$.

Condition 12. There exists a positive constant $r$ such that

$$
\frac{r}{F_{1}+F_{2} r+F_{3} \psi\left(l r+\|\phi\|_{\mathscr{B}_{h}}+l M|\phi(0)|\right)}>1,
$$

where

$$
\begin{aligned}
F_{1} & =K_{1}+\frac{M M_{2} M_{3} b^{\alpha}}{\Gamma(1+\alpha)} \cdot\left\{\left|x_{1}\right|+M|\phi(0)|+K_{1}\right\}, \\
F_{2} & =K_{2}+\frac{M M_{2} M_{3} b^{\alpha}}{\Gamma(1+\alpha)} \cdot K_{2}, \\
F_{3} & =\left[1+\frac{M M_{2} M_{3} b^{\alpha}}{\Gamma(1+\alpha)}\right] \frac{b^{\alpha} M}{\Gamma(1+\alpha)} \sup _{s \in J} p(s), \\
K_{1} & =M\left[\left\|(-A)^{-\beta}\right\|\left(c_{1}\|\phi\|_{\mathscr{B}_{h}}+c_{2}\right)\right] \\
& +\left[\left\|(-A)^{-\beta}\right\|+\frac{c_{1-\beta} \Gamma(1+\beta)}{\Gamma(1+\alpha \beta)} \cdot \frac{b^{\alpha \beta}}{\beta}\right] \\
& \cdot\left(c_{1}\|\phi\|_{\mathscr{B}_{h}}+c_{1} l M|\phi(0)|+c_{2}\right)+M \sum_{k=1}^{m} d_{k}, \\
K_{2} & =\left\|(-A)^{-\beta}\right\| c_{1} l+\frac{c_{1-\beta} \Gamma(1+\beta)}{\Gamma(1+\alpha \beta)} \cdot \frac{b^{\alpha \beta}}{\beta} c_{1} l .
\end{aligned}
$$

Next theorem includes the condition for exact controllability of system (22) on the interval $J$.

Theorem 13 (see [53]). If the Conditions 7-12 hold, then system (22) is controllable on the interval J.

Based on a fixed point theorem (Theorem 10), sufficient conditions for the exact controllability of the fractional impulsive neutral functional differential inclusions have been obtained.

3.3. Approximate Controllability of Nonlocal Neutral Fractional Integrodifferential Equations with Finite Delay. In paper [59], authors obtain a set of sufficient conditions to prove the approximate controllability for a class of nonlocal neutral fractional integrodifferential equations, with time varying delays, considered in a Hilbert space.
They consider the following equation:

$$
\begin{aligned}
{ }^{c} D^{\alpha} & {[x(t)+h(t, x(t-k(t)))] } \\
& =-A x(t)+I_{t}^{1-\alpha} f(t, x(t-v(t)))+B u(t), \\
t & \in J=[0, b], x(t)=\phi(t)+g(x)(t), t \in[-a, 0],
\end{aligned}
$$

where

(i) $a>0$;

(ii) $\alpha \in(0,1)$;

(iii) $k, v:[0,+\infty) \rightarrow(0, a]$, and $(a>0)$ are continuous functions;

(iv) $f:[0,+\infty) \times X \rightarrow X, h:[0,+\infty) \times X \rightarrow X_{\alpha}$, and $g: \mathscr{C} \rightarrow C([a, 0], X)$ are continuous and nonlinear functions; here $0<q \leq 1, \mathscr{C}:=C([a, b], X)$.

Let $x(b, \phi, u)$ be the state value of (29) at terminal time $b$ corresponding to the initial value and the control function $u$. Define the set $R(b, \phi)=\left\{x(b, \phi, u): u \in L^{2}(J, U)\right\}$, which is called reachable set of the system (29) at time $b$, and its closure in $X$ is denoted by $\overline{R(b, \phi)}$.

Definition 14 (see [59]). The dynamical system (29) is called approximately controllable on $J$ if $\overline{R(b, \phi)}=X$; that is, for given $\varepsilon>0$, however small, it is possible to steer from the point to within a distance $\varepsilon$ from all points in the state space $X$ at time $b$.

Now, we introduce some conditions which will be used in presented results.

\section{Condition 13.}

$$
\begin{aligned}
\varepsilon R\left(\varepsilon, \Gamma_{0}^{b}\right) & \longrightarrow 0 \text { as } \\
\varepsilon & \longrightarrow 0^{+} \text {in strong operator topology. }
\end{aligned}
$$

Condition 14. The function $h: J \times X \rightarrow X_{q}$ satisfies that, for each $x \rightarrow X$, the function $h(\cdot, x)$ is strongly measurable in $X_{\alpha}$ over the interval $J$ and there exists a positive constant $L_{h}$ such that for each $t \in J$

$$
\begin{aligned}
\|h(t, x)-h(t, y)\|_{\alpha} \leq L_{h}\|x-y\|, & \forall x, y \in X, \\
\|h(t, x)\|_{\alpha} \leq L_{h}(1+\|x\|), & \forall x \in X .
\end{aligned}
$$

Condition 15. The function $f: J \times X \rightarrow X$ satisfies the following.

(i) For any $t \in J$, the function $f(t, \cdot): X \rightarrow X$ is continuous, and for all $x \in X$, the function $f(\cdot, x)$ is strongly measurable.

(ii) For each $r>0$, there exist $a_{r}(\cdot) \in L^{1}\left([0, t], R^{+}\right)$and $t \in J$, such that

$\sup \{\|f(t, x)\|:\|x\| \leq r\} \leq a_{r}(t), \quad$ for a.e. $t \in J$,

$$
\lim _{r \rightarrow \infty} \inf \frac{1}{r} \int_{0}^{t} a_{r}(s) d s=\sigma<+\infty .
$$


Condition 16. $g: \mathscr{C} \rightarrow C([-a, 0], X)$ is a continuous function and there exists a positive constant $L_{g}$ such that

$$
\begin{aligned}
&\|g(x)-g(y)\|_{C([-a, 0], X)} \leq L_{g}\|x-y\|_{\mathscr{C}}, \\
& \forall x, y \in \mathscr{C}, \\
&\|g(x)\|_{C([-a, 0], X)} \leq L_{g}\left(1+\|x\|_{\mathscr{C}}\right), \quad \forall x \in \mathscr{C} .
\end{aligned}
$$

For any $\varepsilon>0$ and $z \in X$, we define a control $u_{\varepsilon}(t, x)$ as

$$
\begin{aligned}
& u_{\varepsilon}(t, x)=B^{*} V^{*}(b-t) R\left(\varepsilon, \Gamma_{0}^{b}\right)\{z-U(b)[\phi(0) \\
& +g(x)(0)+h(0, \phi(-k(0))+g(x)(-k(0)))] \\
& +h(b, x(b-k(b)))-\int_{0}^{b}(b-s)^{q-1} A V(b-s) \\
& \quad \times h(s, x(s-k(s))) d s \\
& \left.\quad-\int_{0}^{b} U(b-s) f(s, x(s-v(s))) d s\right\} \\
& V(t)=q \int_{0}^{\infty} \vartheta \psi_{q}(\vartheta) T\left(t^{q} \vartheta\right) d \vartheta
\end{aligned}
$$

where $\psi_{q}(\vartheta)$ satisfies the condition of a probability density function defined on $(0, \infty)$; that is, $\psi_{q}(\vartheta) \geq 0, \int_{0}^{\infty} \psi_{q}(\vartheta) d \vartheta=$ 1 , and $\int_{0}^{\infty} \vartheta \psi_{q}(\vartheta)=1 / \Gamma(1+q) ; B^{*}$ and $V^{*}$ denote the adjoint of $B$ and $V$, respectively.

For the sake of convenience, we introduce the following denotations:

$$
\begin{aligned}
K & =\frac{1}{\varepsilon} \frac{M b^{q}}{\Gamma(q+1)}\|B\| \sup _{0 \leq t \leq b}\left\|B^{*} V^{*}(b-t)\right\|, \\
N_{\alpha} & =\frac{q M_{\alpha} \Gamma(1+\alpha)}{\Gamma(1+q \alpha)}, \\
l= & \frac{1}{\varepsilon}\|B\| \sup _{0 \leq t \leq b}\left\|B^{*} V^{*}(b-t)\right\|[\|z\|+M\{\|\phi(0)\| \\
& +L_{g}\left(1+\|x\|_{\mathscr{C}}\right) \\
& \left.+C_{\alpha} L_{h}\left(1+\|\phi(-k(0))\|+L_{g}\left(1+\|x\|_{\mathscr{C}}\right)\right)\right\} \\
& +C_{\alpha} L_{h}(1+\|x(b-k(b))\|)+N_{\alpha} \frac{b^{q \alpha}}{q \alpha} L_{h}(1 \\
& \left.\left.+\|x\|_{\mathscr{C}}\right)+M \int_{0}^{b} a_{r}(s) d s\right],
\end{aligned}
$$

where $M \geq 1, C_{\alpha}>0, M_{1-\alpha}>0, \alpha>0$, and $M_{\alpha}>0$.
Theorem 15 (see [59]). Assume that the Conditions 14-16 hold. System (29) corresponding to the control $u_{\varepsilon}(t, x)$ has a mild solution for each $\varepsilon>0$ provided that

$$
\begin{aligned}
& (K+1) \\
& \quad\left[M\left(L_{g}+\sigma\right)+L_{h}\left(C_{\alpha}\left(M L_{g}+1\right)+N_{\alpha} \frac{b^{q \alpha}}{q \alpha}\right)\right] \\
& \quad<1 .
\end{aligned}
$$

Theorem 16 (see [59]). Suppose that the Conditions 13, 14, 15, and 16 hold. Besides, one assumes additionally that the functions $f: J \times X \rightarrow X, h: J \times X \rightarrow X_{q}$, and $g: C([a, b], X) \rightarrow$ $C([a, 0], X)$ are bounded and $M L_{g}+L_{h}\left(M L_{g}+1\right) C_{\alpha}<1$. Then the nonlocal neutral fractional integrodifferential equations with finite delay (29) are approximately controllable on J.

Theorem 16 is proved by Krasnoselskii's fixed point theorem.

3.4. Exact Controllability of Fractional Neutral Integrodifferential Systems with State-Dependent Delay in Banach Spaces. In paper [60] the authors execute Banach contraction fixed point theorem combined with resolvent operator to analyze the exact controllability results for fractional neutral integrodifferential systems with state-dependent delay in Banach spaces. Motivation to do it implies from their papers [61-63]. In article [60] they study the controllability of mild solutions for a fractional neutral integrodifferential system with statedependent delay of the model

$$
\begin{aligned}
& D_{t}^{\alpha}\left[x(t)+G\left(t, x_{\varrho\left(t, x_{t}\right)}, \int_{0}^{t} e_{1}\left(t, s, x_{\varrho\left(s, x_{s}\right)}\right) d s\right)\right] \\
& =A x(t)+\int_{0}^{t} B(t-s) x(s) d s \\
& \quad+F\left(t, x_{Q\left(t, x_{t}\right)}, \int_{0}^{t} e_{2}\left(t, s, x_{\varrho\left(s, x_{s}\right)}\right) d s\right)+C u(t), \\
& t \in J=[0, T], x_{0}=\varsigma(t) \in \mathscr{B}_{h}, x^{\prime}(0)=0, t \in(-\infty, 0],
\end{aligned}
$$

where

(i) $x(\cdot)$ is unknown and needs values in the Banach space $X$ having norm $\|\cdot\|$;

(ii) $\alpha \in(1,2)$;

(iii) $A$ and $(B(t))_{t \geq 0}$ are closed linear operators described on a regular domain which is dense in $(X,\|\cdot\|)$;

(iv) $C$ is a bounded linear operator from $U$ to $X$;

(v) $G,: J \times \mathscr{B}_{h} \times X \rightarrow X, e_{i}: \mathscr{D} \times \mathscr{B}_{h} \rightarrow X, i=1,2$; $\mathscr{D}=\{(t, s) \in J \times J: 0 \leq s \leq t \leq T\}$, and $\varrho: J \times \mathscr{B}_{h} \rightarrow$ $(-\infty, T]$ are apposite functions.

If $x:(-\infty, T] \rightarrow X, T>0$, is continuous on $J$ and $x_{0} \in \mathscr{B}_{h}$, then for every $t \in J$ the accompanying conditions hold.

(1) $x_{t}$ is $\mathscr{B}_{h}$.

(2) $\|x(t)\|_{X} \leq H\left\|x_{t}\right\|_{\mathscr{B}_{h}}$. 
(3) $\left\|x_{t}\right\|_{\mathscr{B}_{h}} \leq \mathscr{D}_{1}(t) \sup \left\{\|x(s)\|_{X}: 0 \leq s \leq t\right\}+$ $\mathscr{D}_{2}(t)\left\|x_{0}\right\|_{\mathscr{B}_{h}}$, where $H>0$ is a constant and $\mathscr{D}_{1}(\cdot)$ : $[0,+\infty) \rightarrow[0,+\infty)$ is continuous, $\mathscr{D}_{2}(\cdot):[0,+\infty) \rightarrow$ $[0,+\infty)$ is locally bounded, and $\mathscr{D}_{1}$ and $\mathscr{D}_{2}$ are independent of $x(\cdot)$.

(4) The function $t \rightarrow \varsigma_{t}$ is well described and continuous from the set

$$
R\left(\varrho^{-}\right)=\left\{\varrho(s, \varsigma):(s, \varsigma) \in[0, T] \times \mathscr{B}_{h}\right\}
$$

into $\mathscr{B}_{h}$ and there is a continuous and bounded function $J^{\varsigma}: R\left(\varrho^{-}\right) \rightarrow(0, \infty)$ to ensure that $\left\|\varsigma_{t}\right\|_{\mathscr{B}_{h}} \leq$ $J^{\varsigma}(t)\left\|_{\varsigma}\right\|_{\mathscr{B}_{h}}$ for every $t \in R\left(\varrho^{-}\right)$.

Recognize the space

$$
\begin{aligned}
\mathscr{B}_{T} & =\{x:(-\infty, T] \\
& \left.\longrightarrow X:\left.x\right|_{J} \text { is continuous and } x_{0} \in \mathscr{B}_{h}\right\},
\end{aligned}
$$

where $\left.x\right|_{J}$ is the constraint of $x$ to the real compact interval on $J$. The function $\|\cdot\|_{\mathscr{B}_{T}}$ to be a seminorm in $\mathscr{B}_{T}$ is described by

$$
\begin{aligned}
& \|x\|_{\mathscr{B}_{T}}=\|\varsigma\|_{\mathscr{B}_{h}}+\sup \left\{\|x(s)\|_{X}: s \in[0, T]\right\}, \\
& x \in \mathscr{B}_{T} .
\end{aligned}
$$

Definition 17. Let $x_{T}(\varsigma ; u)$ be the state value of model (37) at terminal time $T$ corresponding to the control $u$ and the initial value $\varsigma \in \mathscr{B}_{h}$. Present the set $\mathscr{R}(T, \varsigma)=\left\{x_{T}(\varsigma ; u)(0): u(\cdot) \epsilon\right.$ $\left.L^{2}(J, U)\right\}$, which is known as the reachable set of model (37) at terminal time $T$.

Definition 18. Model (37) is said to be exactly controllable on $\mathscr{J}$ if $\mathbb{R}(T ; \varsigma)=X$.

Now, according to the article [60] we will present the exact controllability results for the structure (37) under Banach fixed point theorem. First of all, we present the mild solution for model (37).

Definition 19 ([64], Definition 3.4). A function $x$ : $(-\infty, T] \rightarrow X$ is called a mild solution of $(37)$ on $[0, T]$, if $x_{0}=\varsigma ;\left.x\right|_{[0, T]} \in C([0, T]: X)$; the function

$$
\begin{aligned}
s \longrightarrow & A S_{\alpha}(t-s) G\left(s, x_{\varrho\left(s, x_{s}\right)}, \int_{0}^{s} e_{1}\left(s, \tau, x_{\varrho\left(\tau, x_{\tau}\right)}\right) d \tau\right) \\
s \longrightarrow & \int_{0}^{s} B(s-\tau) S_{\alpha}(t-s) \\
& \cdot G\left(\tau, x_{\varrho\left(\tau, x_{\tau}\right)}, \int_{0}^{\tau} e_{1}\left(\tau, \varepsilon, x_{\varrho\left(\varepsilon, x_{\tau}\right)}\right) d \varepsilon\right) d \tau
\end{aligned}
$$

is integrable on $[0, t)$ for all $t \in(0, T]$ and for $t \in[0, T]$;

$$
\begin{aligned}
& x(t)=R_{\alpha}(t)[\varsigma(0)+G(0, \varsigma(0), 0)] \\
& -G\left(t, x_{\varrho\left(t, x_{t}\right)}, \int_{0}^{t} e_{1}\left(t, s, x_{\varrho\left(s, x_{s}\right)}\right) d s\right)-\int_{0}^{t} A S_{\alpha}(t \\
& -s) G\left(s, x_{\varrho\left(s, x_{s}\right)}, \int_{0}^{s} e_{1}\left(s, \tau, x_{\varrho\left(\tau, x_{\tau}\right)}\right) d \tau\right) d s \\
& -\int_{0}^{t} \int_{0}^{s} B(s-\tau) S_{\alpha}(t-s) \\
& \cdot G\left(\tau, x_{\varrho\left(\tau, x_{\tau}\right)}, \int_{0}^{\tau} e_{1}\left(\tau, \varepsilon, x_{\varrho\left(\varepsilon, x_{\varepsilon}\right)}\right) d \varepsilon\right) d \tau d s \\
& +\int_{0}^{t} S_{\alpha}(t-s) \\
& \quad+F\left(s, x_{\varrho\left(s, x_{s}\right)}, \int_{0}^{s} e_{2}\left(s, \tau, x_{\varrho\left(\tau, x_{\tau}\right)}\right) d \tau\right) d s \\
& +\int_{0}^{t} S_{\alpha}(t-s) C u(s) d s .
\end{aligned}
$$

Presently, we itemizing the subsequent conditions.

Condition 17. The operator families $R_{\alpha}(t)$ and $S_{\alpha}(t)$ are compact for all $t>0$, and there exists a constant $M$ in a way that $\left\|R_{\alpha}(t)\right\|_{\mathscr{L}(X)} \leq M$ and $\left\|S_{\alpha}(t)\right\|_{\mathscr{L}(\mathbb{X})} \leq M$ for every $t \in J$ and

$$
\left\|(-A)^{9} S_{\alpha}(t)\right\|_{X} \leq M t^{\alpha(1-9)-1}, \quad 0<t \leq T,
$$

where $\mathscr{L}(X)$ symbolizes the Banach space of all bounded linear operators from $X$ into $X$ endowed with the uniform operator topology, having its norm recognized as $\|\cdot\|_{\mathscr{L}(X)}$.

Condition 18. The subsequent conditions are fulfilled.

(a) $B(\cdot) x \in C(J, X)$ for every $x \in\left[D\left((-A)^{1-9}\right)\right]$.

(b) There is a function $\mu(\cdot) \in L^{1}\left(J, \mathbb{R}^{+}\right)$, to ensure that

$$
\begin{aligned}
\left\|B(s) S_{\alpha}(t)\right\|_{\mathscr{L}\left(\left[D\left((-A)^{9}\right)\right], X\right)} \leq M \mu(s) t^{\alpha \vartheta-1} & \\
& \\
& 0 \leq s<t \leq T .
\end{aligned}
$$

Condition 19. The function $F: J \times \mathscr{B}_{h} \times X \rightarrow X$ is continuous and one can find positive constants $L_{\mathscr{F}}, \widetilde{L}_{\mathscr{F}}$, and $L_{\mathscr{F}}^{*}>0$ in ways that, for all $t \in J$ and $x, y \in X$,

$$
\begin{aligned}
& \left\|F\left(t, \psi_{1}, x\right)-F\left(t, \psi_{2}, y\right)\right\|_{X} \\
& \quad \leq L_{\mathscr{F}}\left\|\psi_{1}-\psi_{2}\right\|_{\mathscr{B}_{h}}+\widetilde{L}_{F}\|x-y\|_{X}, \\
& L_{\mathscr{F}}^{*}=\max _{t \in J}\|F(t, 0,0)\|_{X} .
\end{aligned}
$$


Condition 20. $e_{i}: \mathscr{D} \times \mathscr{B}_{h} \rightarrow X$ is continuous and one can find constants $L_{e_{i}}>0$ and $L_{e_{i}}^{*}>0$ to ensure that, for all $(t, s) \in$ $\mathscr{D}$ and $(\varsigma, \psi) \in \mathscr{B}_{h}^{2}, i=1,2$;

$$
\begin{aligned}
\left\|e_{i}(t, s, \varsigma)-e_{i}(t, s, \psi)\right\|_{X} \leq L_{e_{i}}\|\varsigma-\psi\|_{\mathscr{B}_{h}}, & \\
L_{e_{i}}^{*}=\max _{t \in J}\left\|e_{i}(t, s, 0)\right\|_{X}, & \\
& i=1,2 .
\end{aligned}
$$

Condition 21. The function $G(\cdot)$ is $(-A)^{9}$-values; $G$ : $J \times$ $\mathscr{B}_{h} \times \mathbb{X} \rightarrow\left[D\left((-A)^{-\vartheta}\right)\right]$ is continuous and there exist positive constants $L_{G}, \widetilde{L}_{G}>0$ and $L_{G}^{*}>0$ such that, for all $\left(t, \varsigma_{j}\right) \in$ $J \times \mathscr{B}_{h}, j=1,2 ; x, y \in X$,

$$
\begin{gathered}
\left\|(-A)^{\vartheta} G\left(t, \varsigma_{1}, x\right)-(-A)^{\vartheta} G\left(t, \varsigma_{2}, y\right)\right\|_{X} \\
\leq L_{G}\left\|\varsigma_{1}-\varsigma_{2}\right\|_{\mathscr{B}_{h}}+\widetilde{L}_{G}\|x-y\|_{X}, \\
\left\|(-A)^{\vartheta} G(t, \varsigma, 0)\right\|_{X} \leq L_{G}\|\varsigma\|_{\mathscr{B}_{h}}+L_{G}^{*},
\end{gathered}
$$

where

$$
L_{G}^{*}=\max _{t \in J}\left\|(-A)^{\vartheta} G(t, 0,0)\right\|_{X}
$$

Condition 22. The following inequalities hold.

(i) Let

$$
\begin{aligned}
& \left(\frac{1}{\gamma} M^{2} M_{\mathscr{C}}^{2} T\right)\left\|x_{T}\right\|+\left(1+\frac{1}{\gamma} M^{2} M_{\mathscr{C}}^{2} T\right) \\
& \quad \cdot\left[M M_{0} L_{G}\|s\|_{\mathscr{B}_{h}}+M M_{0} L_{G}^{*}+\left(L_{G}^{*}+\widetilde{L}_{G} T L_{e_{1}}^{*}\right)\right. \\
& \quad \cdot\left\{M_{0}+\frac{M T^{\alpha \vartheta}}{\alpha \vartheta}\left(1+\int_{0}^{T} \mu(\tau) d \tau\right)\right\}+M T\left(L_{\mathscr{F}}^{*}\right. \\
& \left.\quad+\widetilde{L}_{\mathscr{F}} T L_{e_{2}}^{*}\right)+\left(\mathscr{D}_{1}^{*} r+c_{n}\right)\left[M T\left(L_{\mathscr{F}}+\widetilde{L}_{\mathscr{F}} T L_{e_{2}}\right)\right. \\
& \quad+\left\{M_{0}+\frac{M T^{\alpha \vartheta}}{\alpha \vartheta}\left(1+\int_{0}^{T} \mu(\tau) d \tau\right)\right\} \\
& \left.\quad \cdot\left(L_{G}+\widetilde{L}_{G} T L_{e_{1}}\right)\right] \leq r,
\end{aligned}
$$

for some $r, \gamma>0$.

(ii) Let

$$
\begin{aligned}
\Lambda & =\left(1+\frac{1}{\gamma} M^{2} M_{\mathscr{C}}^{2} T\right) \mathscr{D}_{1}^{*}\left[M T\left(L_{\mathscr{F}}+\widetilde{L}_{\mathscr{F}} T L_{e_{2}}\right)\right. \\
& +\left\{M_{0}+\frac{M T^{\alpha \vartheta}}{\alpha \vartheta}\left(1+\int_{0}^{T} \mu(\tau) d \tau\right)\right\} \\
& \left.\cdot\left(L_{G}+\widetilde{L}_{G} T L_{\mathrm{e}_{1}}\right)\right]<1
\end{aligned}
$$

be such that $0 \leq \Lambda<1$.
Theorem 20 (see [60]). Assume that the Conditions 17-22 hold. Then, control system (37) is exactly controllable on J.

Proof of the Theorem 20 is based on contraction mapping principle [60].

3.5. Controllability for a Class of Fractional Neutral Integrodifferential Equations with Unbounded Delay. The paper [65] focuses on establishing the sufficient conditions for the exact controllability for a class of fractional neutral integrodifferential equations with infinite delay in Banach spaces formulated as follows:

$$
\begin{aligned}
& { }^{c} D_{t}^{\alpha}\left(x(t)+f\left(t, x_{t}\right)\right) \\
& =A x(t)+\int_{0}^{t} G(t-s) x(s) d s+(B u)(t) \\
& +g\left(t, x_{t}\right), \\
& \quad t \in I=[0, b], x_{0}=\phi \in \mathscr{B}_{h}, x^{\prime}(0)=x_{1},
\end{aligned}
$$

where

(i) $\alpha \in(1,2)$;

(ii) $A, G(t)$, for $t \geqslant 0$, are closed linear operators defined on a common domain $\mathscr{D}=D(A)$ which is dense in $X$;

(iii) $f, g:[0, b] \times \mathscr{B}_{h} \rightarrow X$ are appropriate functions.

Some necessary notations for the above-mentioned system were presented in Basic Notations Section. The other ones are as follows.

(i) $[D(A)]$ is the domain of $A$ endowed with the graph norm.

(ii) $\left(Z,\|\cdot\|_{Z}\right)$ and $\left(W,\|\cdot\|_{W}\right)$ are Banach spaces.

(iii) $\mathscr{L}(Z, W)$ stands for the Banach space of bounded linear operators from $Z$ into $W$ endowed with the uniform operator topology. When $Z=W$ then we will write $\mathscr{L}(Z)$.

(iv) $\widehat{K}$ denotes the Laplace transform of $K$ for appropriate functions $K:[0, \infty) \rightarrow Z$.

(v) $\|x\|_{Z, b}=\sup \left\{\|x(s)\|_{Z}: s \in[0, b]\right\}$ for a bounded function $x:[0, a] \rightarrow Z$ and $b \in[0, a]$; shortly we will write $\|x\|_{b}$ when no confusion about the space $Z$ arises.

In [65] the contraction mapping principle is used to formulate and prove conditions for exact controllability for the system (51). To obtain the exact controllability result the following lemmas and conditions were made [65].

Lemma 21. One can assume there exists $M>0$ such that $\left\|R_{\alpha}(t)\right\| \leqslant M$ and $\left\|S_{\alpha}(t)\right\| \leqslant M$ for all $t \in[0, b]$. Additionally, $M_{b}=\sup _{s \in[0, b]} M(s)$ and $K_{b}=\sup _{s \in[0, b]} K(s)$ are the constants. Moreover $N_{(-A)^{9} f}, N_{f}, N_{g}$ represent the supreme of the functions $(-A)^{9} f, f$ and $g$ on $[0, b] \times B_{r}\left[0, \mathscr{B}_{h}\right]$, respectively. 
Lemma 22 (see [66]). There exists a constant $C$ such that

$$
\left\|(-A)^{9}\right\| \leqslant C \quad \text { for } 0 \leqslant \vartheta \leqslant 1 .
$$

Condition 23. The given conditions hold.

(i) $G(\cdot) x \in C(I, X)$ for every $x \in\left[D\left((-A)^{1-9}\right)\right]$.

(ii) There is function $\mu(\cdot) \in L^{1}\left(I ; \mathbb{R}^{+}\right)$, such that $\left\|G(s) S_{\alpha}(t)\right\|_{\mathscr{L}\left(\left[D\left((-A)^{9}\right)\right], X\right)} \leqslant M \mu(s) t^{\alpha 9-1}, 0 \leqslant s<t \leqslant b$.

Condition 24. The function $f(\cdot)$ is $(-A)^{9}$-valued, $f: I \times$ $\mathscr{B}_{h} \rightarrow\left[D\left((-A)^{-9}\right)\right]$, the function $g(\cdot)$ is defined on $g$ : $I \times \mathscr{B}_{h} \rightarrow X$, and there exist positive constants $L_{f}$ and $L_{g}$ such that for all $\left(t_{i}, \psi_{j}\right) \in I \times \mathscr{B}_{h}$ the following inequalities are satisfied

$$
\begin{aligned}
& \left\|(-A)^{9} f\left(t_{1}, \psi_{1}\right)-(-A)^{9} f\left(t_{2}, \psi_{2}\right)\right\| \\
& \leqslant L_{f}\left(\left|t_{1}-t_{2}\right|+\left\|\psi_{1}-\psi_{2}\right\|_{\mathscr{B}_{h}}\right), \\
& \left\|g\left(t_{1}, \psi_{1}\right)-g\left(t_{2}, \psi_{2}\right)\right\| \\
& \quad \leqslant L_{g}\left(\left|t_{1}-t_{2}\right|+\left\|\psi_{1}-\psi_{2}\right\|_{\mathscr{B}_{h}}\right) .
\end{aligned}
$$

Condition 25. The linear fractional control system defined as

$$
\begin{aligned}
{ }^{c} D_{t}^{\alpha} x(t) & =A x(t)+(B u)(t), \\
x(0) & =x_{0}, \\
x^{\prime}(0) & =0
\end{aligned}
$$

is exactly controllable.

In the next theorem we present conditions for exact controllability for the system (51).

Theorem 23 (see [65]). If Conditions 23-25 and

$$
\begin{aligned}
& \left(1+\frac{1}{\gamma} M^{2} M_{B}^{2} b\right) K_{b} \cdot\left[M\left(H\|\phi\|_{\mathscr{B}_{h}}+N_{f}\right)+N_{f}\right. \\
& +N_{(-A)^{9} f} M \frac{b^{\alpha \vartheta}}{\alpha \vartheta}+N_{(-A)^{9} f} M \frac{b^{\alpha \vartheta}}{\alpha \vartheta} \int_{0}^{b} \mu(\xi) d \xi \\
& \left.+N_{g} M b\right]<r, \\
& \left(1+\frac{1}{\gamma} M^{2} M_{B}^{2}\right) K_{b} \\
& \quad \cdot\left(L_{f}\left(\left\|(-A)^{-\vartheta}\right\|+\frac{M b^{\alpha \vartheta}}{\alpha \vartheta}+\frac{M b^{\alpha \vartheta}}{\alpha \vartheta} \int_{0}^{b} \mu(\xi) d \xi\right)\right. \\
& \left.+M L_{g} b\right)<1
\end{aligned}
$$

are satisfied, then control system (51) is exactly controllable on I.
Theorem 23 is proved in [65] by using the contraction mapping.

Additionally, the authors of paper [65] study the exact controllability of the fractional neutral integrodifferential system with nonlocal condition of the following form:

$$
\begin{aligned}
& { }^{c} D_{t}^{\alpha}\left(x(t)+f\left(t, x_{t}\right)\right) \\
& \quad=A x(t)+\int_{0}^{t} G(t-s) x(s) d s+(B u)(t)+g\left(t, x_{t}\right), \\
& t \in I=[0, b], x_{0}=\varphi+q\left(x_{t_{1}}, x_{t_{2}}, \ldots, x_{t_{n}}\right) \in \mathscr{B}_{h}, x^{\prime}(0)=x_{1},
\end{aligned}
$$

where $0<t_{1}<t_{2}<\cdots<t_{n} \leqslant b ; q: \mathscr{B}_{h}^{n} \rightarrow \mathscr{B}_{h}$ is given function such that the next condition holds.

Condition 26. The function $q: \mathscr{B}_{h}^{n} \rightarrow \mathscr{B}_{h}$ is continuous and there exist positive constants $L_{i}(q)$ such that

$$
\begin{aligned}
& \left\|q\left(\psi_{1}, \psi_{2}, \ldots, \psi_{n}\right)-q\left(\varphi_{1}, \varphi_{2}, \ldots, \varphi_{n}\right)\right\| \\
& \quad \leqslant \sum_{i=1}^{n} L_{i}(q)\left\|\psi_{i}-\varphi_{i}\right\|_{\mathscr{B}_{h}}
\end{aligned}
$$

for every $\psi_{i}, \varphi_{i} \in B_{r}\left[0, \mathscr{B}_{h}\right]$ and assume $N_{q}=\sup \left\{\| q\left(\psi_{t_{1}}\right.\right.$, $\left.\left.\psi_{t_{2}}, \ldots, \psi_{t_{n}}\right) \|: \psi_{i} \in B_{r}\left[0, \mathscr{B}_{h}\right]\right\}$.

The next theorem includes the required conditions for system (57) to be exactly controllable.

Theorem 24 (see [65]). Assume that the conditions of Theorem 23 are satisfied. Further, if Condition 26 is satisfied, then fractional system (57) is exactly controllable on I provided that

$$
\begin{aligned}
(1 & \left.+\frac{1}{\gamma} M^{2} M_{B}^{2} b\right)\left(\left(M_{b}+K_{b} M H\right)\|\varphi\|_{\mathscr{B}_{h}}+\left(M_{b}\right.\right. \\
& \left.+K_{b} M\right) N_{q}+K_{b}(M+1) N_{f}+K_{b} N_{(-A)^{9} f} M \\
& \left.\cdot \frac{b^{\alpha \vartheta}}{\alpha \vartheta}\left(1+\int_{0}^{b} \mu(\xi) d(\xi)\right)+K_{b} N_{g} M b\right)<r \\
\Lambda & =\max \left\{M_{b}\left(M_{b} \sum_{i=1}^{n} L_{i}(q)+K_{b} \theta\right)\right. \\
& \left.K_{b}\left(M_{b} \sum_{i=1}^{n} L_{i}(q)+K_{b} \theta\right)\right\}<1,
\end{aligned}
$$

where

$$
\begin{aligned}
\theta & =\left(1+\frac{1}{\gamma} M^{2} M_{B}^{2} b\right)\left(M \sum_{i=1}^{n} L_{i}(q)\right. \\
& +L_{f}\left(\left\|(-A)^{-9}\right\|+\frac{M b^{\alpha \vartheta}}{\alpha \vartheta}+\frac{M b^{\alpha \vartheta}}{\alpha \vartheta} \int_{0}^{b} \mu(\xi) d(\xi)\right) \\
& \left.+M L_{g} b\right) .
\end{aligned}
$$

As before, the proof of Theorem 24 is led by contraction mapping. 
3.6. Controllability of Neutral Fractional Functional Equations with Impulses and Infinite Delay. Authors of [67] investigate the exact controllability of a class of fractional order neutral integrodifferential equations with impulses and infinite delay in the following form:

$$
\begin{aligned}
{ }^{c} D_{t}^{\alpha}\left[x(t)+g\left(t, x_{t}\right)\right]=A\left[x(t)+g\left(t, x_{t}\right)\right]+J_{t}^{1-\alpha}\left[B u(t)+f\left(t, x_{t}, H x(t)\right)\right], \\
t \in J=[0, b], t \neq t_{k}, \Delta x\left(t_{k}\right)=I_{k}\left(x\left(t_{k}^{-}\right)\right), k=1,2, \ldots, m, x_{0}=\phi \in \mathscr{B}_{h},
\end{aligned}
$$

where

(i) $0<\alpha<1$;

(ii) $g: J \times \mathscr{B}_{h} \times X \rightarrow X$ are given functions;

(iii) $H x(t)=\int_{0}^{t} G(t, s) x(s) d s$, where $G \in C\left(D, R^{+}\right)$is the set of all positive continuous functions on $D=$ $\left\{(t, s) \in R^{2}: 0 \leqslant s \leqslant t \leqslant b\right\} ;$

(iv) $x_{b}(\phi ; u)$ is the state value.

To formulate a set of sufficient conditions for exact controllability of system (61) next conditions are necessary [67].

Condition 27. There exists a constant $M>0$ such that

$$
\left\|S_{\alpha}(t)\right\|_{L(X)} \leqslant M, \quad \forall t \in[0, b] .
$$

Condition 28. The function $g: J \times \mathscr{B}_{h} \rightarrow X$ is continuous, and there exists a constant $L_{g}>0$ such that

$$
\begin{aligned}
& \left\|g\left(t_{1}, \psi_{1}\right)-g\left(t_{2}, \psi_{2}\right)\right\|_{X} \\
& \leqslant L_{g}\left(\left|t_{1}-t_{2}\right|+\left\|\psi_{1}-\psi_{2}\right\|_{\mathscr{B}_{h}}\right), \\
& \quad t_{i} \in J, \psi_{i} \in \mathscr{B}_{h}, i=1,2 .
\end{aligned}
$$

Condition 29. There exist constants $\mu_{1}>0$ and $\mu_{2}>0$ such that

$$
\begin{aligned}
& \|f(t, \varphi, x)-f(t, \psi, y)\|_{X} \\
& \leqslant \mu_{1}\|\varphi-\psi\|_{\mathscr{B}_{h}}+\mu_{2}\|x-y\|_{X}, \\
& \quad t \in J, \varphi, \psi \in \mathscr{B}_{h}, x, y \in X .
\end{aligned}
$$

Condition 30. $I_{k} \in C(X, X)$, and there exist constants $\rho>0$ such that

$$
\begin{aligned}
\left\|I_{k}(x)-I_{k}(y)\right\|_{X} \leqslant & \rho\|x-y\|_{X}, \\
& x, y \in X, \text { for each } k=1, \ldots, m .
\end{aligned}
$$

Conditions for exact controllability of the fractional impulsive system (61) on $J$ are the content of the next theorem.

Theorem 25 (see [67]). If the Conditions 25 and 27-30 are satisfied and there exists $\gamma>0$, then fractional impulsive system (61) is exactly controllable on J provided that

$$
\begin{aligned}
\widehat{L} & =\left(1+\frac{1}{\gamma} M_{B}^{2} M^{2}\right)\left[m M \rho+m M L_{g} C_{1}(2+\rho)\right. \\
& \left.+L_{g} C_{1}+M b\left(\mu_{1} C_{1}+\mu_{2} H\right)\right]<1,
\end{aligned}
$$

where $C_{1}=\sup _{0<\tau<b} C_{1}(\tau)$ and $H=\sup _{t \in[0, b]} \int_{0}^{t} G(t, s) d s<$ $\infty$.

Moreover, in paper [67], the approximate controllability of system (61) was discussed too and the results are presented below.

Theorem 26 (see [67]). Assume that Conditions 27-30 hold and that the family $\left\{S_{\alpha}(t): t>0\right\}$ is compact. In addition, assume that the function $f$ is uniformly bounded and the linear system (54) associated with the system (61) is approximately controllable; then the nonlinear fractional control system with infinite delay (61) is approximately controllable on $[0, b]$.

Theorems 25 and 26 are proved in [67] by contraction mapping theorem.

3.7. Controllability for a Class of Fractional Order Neutral Evolution Control Systems. In [68], authors study the exact controllability of fractional control systems with states and controls in Hilbert spaces. Their investigations were started from fractional nonlinear neutral functional differential equation described as follows:

$$
\begin{array}{r}
{ }^{C} D_{t}^{\alpha}\left[x(t)-h\left(t, x_{t}\right)\right]=A x(t)+B u(t)+f\left(t, x_{t}\right), \\
t \in J=[0, b], x_{0}(\vartheta)=\phi_{\vartheta}, \vartheta \in[-r, 0] .
\end{array}
$$

Some necessary notations for the above-mentioned system were presented in Basic Notations Section. The other ones are as follows.

(i) $f, h:[0, \infty) \times C \rightarrow H$ are given functions satisfying certain assumptions.

(ii) $\phi \in C$.

(iii) $x_{t}(\vartheta)=x(t+\vartheta)$, for $\vartheta \in[-r, 0]$.

Next conditions [68] are necessary to present conditions for exact controllability for the nonlinear fractional control system (67) by using the contraction mapping principle.

Condition 31. For each $t \in[0, b]$, the function $f(t, \cdot): C \rightarrow$ $H$ is continuous and for each $x \in C$, the function $f(\cdot, x)$ : $[0, b] \rightarrow H$ is strongly measurable.

Condition 32. There exists a constant $\alpha_{1} \in[0, \alpha]$ and $m \in$ $L^{1 / \alpha_{1}}\left([0, b], R^{+}\right)$such that $|f(t, x)| \leqslant m(t)$ for all $x \in C$ and almost all $t \in[0, b]$. 
Condition 33. The function $h:[0, b] \times C \rightarrow H$ is continuous and there exists a constant $\beta \in(0,1)$ and $H, H_{1}>0$ such that $h \in D\left(A^{\beta}\right)$ and for any $x, y \in C$, the function $A^{\beta} h(\cdot, x)$ is strongly measurable and $A^{\beta} h(t, x)$ satisfies the Lipschitz condition

$$
\left|A^{\beta} h(t, x)-A^{\beta} h(t, y)\right| \leqslant H\|x-y\|
$$

and the inequality

$$
\left|A^{\beta} h(t, x)\right| \leqslant H_{1}(\|x\|+1) .
$$

Condition 34. There exists a constant $\alpha_{2} \in[0, \alpha]$ and $\rho \in$ $L^{1 / \alpha_{2}}\left([0, b], R^{+}\right)$such that

$$
|f(t, x)-f(t, y)| \leqslant \rho(t)\|x-y\|
$$

for any $x, y \in C([-r, b], H)$.

Then, the following theorem is true.

Theorem 27 (see [68]). If Conditions 25 and 31-34 are satisfied, then control system (67) is exactly controllable on $J$ provided that

$$
\begin{aligned}
& {\left[(N+1)\left|A^{-\beta}\right| H+\frac{\Gamma(1+\beta) C_{1-\beta} H b^{\alpha \beta}}{\beta \Gamma(1+\alpha \beta)}\right.} \\
& \left.+\frac{\alpha N N_{2} b^{\left(1+a^{\prime}\right)\left(1-\alpha_{2}\right)}}{\Gamma(1+\alpha)\left(1+a^{\prime}\right)^{1-\alpha_{2}}}\right]\left(\frac{N^{2} M_{B}^{2} N_{2} \alpha b^{\left(1+a^{\prime}\right)\left(1-\alpha_{2}\right)}}{\gamma \Gamma(1+\alpha)\left(1+a^{\prime}\right)^{1-\alpha_{2}}}\right. \\
& +1)<1,
\end{aligned}
$$

where

$$
\begin{aligned}
N & =\sup _{t \in[0, \infty)}|T(t)| \geq 1, \\
\left|A^{\eta} T(t)\right| & \leq \frac{C_{\eta}}{t^{\eta}}, \quad C_{\eta}>0, \eta \in(0,1], 0<t \leq b, \\
N_{1} & =\|m\|_{L^{1 / \alpha_{1}} \in[0, b]}, \\
N_{2} & =\|\rho\|_{L^{1 / \alpha_{2}} \in[0, b]}, \\
a^{\prime} & =\frac{\alpha-1}{1-\alpha_{2}} \in(-1,0) .
\end{aligned}
$$

$T(t)$ is an analytic semigroup.

Moreover, the authors of [68] investigated the exact controllability of system (67) with nonlocal condition defined in the following way:

$$
x_{0}(\vartheta)+\left(g\left(x_{t_{1}}, \ldots, x_{t_{n}}\right)\right)(\vartheta)=\phi(\vartheta), \quad \vartheta \in[-r, 0]
$$

where $g: C^{n} \rightarrow C$ are given functions.

Additionally the authors assumed that function $g$ satisfies the below-presented conditions.
Condition 35. There exists a constant $L>0$ such that

$$
\left\|g\left(x_{t_{1}}, \ldots, x_{t_{n}}\right)-g\left(y_{t_{1}}, \ldots, y_{t_{n}}\right)\right\| \leqslant L\|x-y\|
$$

for $x, y \in C([-r, b], H)$.

\section{Condition 36.}

$$
\begin{aligned}
& {\left[N L+(N+1)\left|A^{-\beta}\right| H+\frac{\Gamma(1+\beta) C_{1-\beta} H b^{\alpha \beta}}{\beta \Gamma(1+\alpha \beta)}\right.} \\
& \left.+\frac{\alpha N N_{2} b^{\left(1+a^{\prime}\right)\left(1-\alpha_{2}\right)}}{\Gamma(1+\alpha)\left(1+a^{\prime}\right)^{1-\alpha_{2}}}\right] \\
& \quad \cdot\left(\frac{N^{2} M_{B}^{2} N_{2} \alpha b^{\left(1+a^{\prime}\right)\left(1-\alpha_{2}\right)}}{\gamma \Gamma(1+\alpha)\left(1+a^{\prime}\right)^{1-\alpha_{2}}}+1\right)<1 .
\end{aligned}
$$

Necessary conditions for the controllability of nonlinear systems are established in the following theorem.

Theorem 28 (see [68]). If the conditions of Theorem 27 and Conditions 35 and 36 are satisfied, then fractional system (67) with nonlocal condition (73) is exactly controllable on J.

Theorems 27 and 28 are proved by contraction mapping theorem.

\section{Conclusions}

The presented paper focuses on the controllability problem of different types of dynamical systems described with fractional order equation. Precisely, the paper presents the results for the selected works from the scope of the investigated controllability of fractional semilinear dynamical systems. Generally speaking, at the beginning, we prove that the semilinear system is controllable if the associated linear system is controllable, too. Next, we pose some conditions for the semilinear dynamical system. The main role is the assumption about Lipschitz continuity. After scrutinizing we observed a research methodology, which is used to solve the controllability problem, not only approximately but also exactly. Below is presented the methodology resulting from indepth analysis of the papers concerning the controllability of nonlinear systems:

(1) Showing a mathematical model of dynamical system

(2) Formulation of the assumptions concerning dynamical systems

(3) Proof of solution existence of state space equation using the fixed point theorem or generally fixed point technique

(4) Proposition of a control transferring the initial state to some neighbourhood of final state

(5) Formulation theorem containing necessary conditions of controllability

(6) Proof of the above-mentioned theorem 
The controllability problems for dynamical systems require the application of various mathematical concepts and methods taken directly from differential geometry, functional analysis, topology, and matrix analysis. It should be noticed that there are many unsolved problems for controllability concepts for different types of dynamical systems. The methodology presented in this paper may well be used in a research on controllability of stochastic dynamical systems [69], in a search of optimal control [70, 71], for systems with constraints on control signal [11], and for dynamical systems with delay in state and control $[12,72]$.

\section{Competing Interests}

The authors declare that there is no conflict of interests regarding the publication of this paper.

\section{Acknowledgments}

The research presented here was done by authors as parts of the projects funded by the National Science Centre granted according to Decisions DEC 2014/13/B/ST7/00755 and DEC 2012/07/B/ST7/01404, respectively.

\section{References}

[1] R. Zawiski, "On controllability and measures of noncompactness," in Proceedings of the 10th International Conference on Mathematical Problems in Engineering, Aerospace and Sciences (ICNPAA '14), vol. 1637, pp. 1241-1246, Narvik, Norway, July 2014.

[2] W. Paszke, P. Dabkowski, E. Rogers, and K. Gałkowski, "New results on strong practical stability and stabilization of discrete linear repetitive processes," Systems \& Control Letters, vol. 77, pp. 22-29, 2015.

[3] J. Wang, Z. Fan, and Y. Zhou, "Nonlocal controllability of semilinear dynamic systems with fractional derivative in Banach spaces," Journal of Optimization Theory and Applications, vol. 154, no. 1, pp. 292-302, 2012.

[4] P. Balasubramaniam, V. Vembarasan, and T. Senthilkumar, "Approximate controllability of impulsive fractional integrodifferential systems with nonlocal conditions in Hilbert space," Numerical Functional Analysis and Optimization, vol. 35, no. 2, pp. 177-197, 2014.

[5] J. Liang and H. Yang, "Controllability of fractional integrodifferential evolution equations with nonlocal conditions," Applied Mathematics and Computation, vol. 254, pp. 20-29, 2015.

[6] P. Balasubramaniam and P. Tamilalagan, "Approximate controllability of a class of fractional neutral stochastic integrodifferential inclusions with infinite delay by using Mainardi's function," Applied Mathematics and Computation, vol. 256, pp. 232-246, 2015.

[7] S. Karthikeyan, K. Balachandran, and M. Sathya, "Controllability of nonlinear stochastic systems with multiple time-varying delays in control," International Journal of Applied Mathematics and Computer Science, vol. 25, no. 2, pp. 207-215, 2015.

[8] X. Ding and J. J. Nieto, "Controllability and optimality of linear time-invariant neutral control systems with different fractional orders," Acta Mathematica Scientia, vol. 35, no. 5, pp. 1003-1013, 2015.
[9] Z. Liu and X. Li, "On the exact controllability of impulsive fractional semilinear functional differential inclusions," Asian Journal of Control, vol. 17, no. 5, pp. 1857-1865, 2015.

[10] T. Mur and H. R. Henriquez, "Relative controllability of linear systems of fractional order with delay," Mathematical Control and Related Fields, vol. 5, no. 4, pp. 845-858, 2015.

[11] B. Sikora, "Controllability of time-delay fractional systems with and without constraints," IET Control Theory \& Applications, vol. 10, no. 3, pp. 320-327, 2016.

[12] B. Sikora, "Controllability criteria for timedelay fractional systems with a retarded state," International Journal of Applied Mathematics and Computer Science, vol. 26, no. 3, pp. 521-531, 2016.

[13] J. Klamka and B. Sikora, "New controllability criteria for fractional systems with varying delays," in Theory and Applications of Non-integer Order Systems: 8th Conference on Non-Integer Order Calculus and Its Applications, Zakopane, Poland, vol. 407 of Lecture Notes in Electrical Engineering, pp. 333-344, Springer, Berlin, Germany, 2017.

[14] L. Górniewicz, S. K. Ntouyas, and D. O’Regan, "Controllability of semilinear differential equations and inclusions via semigroup theory in Banach spaces," Reports on Mathematical Physics, vol. 56, no. 3, pp. 437-470, 2005.

[15] X. Fu, "Controllability of non-densely defined functional differential systems in abstract space," Applied Mathematics Letters, vol. 19, no. 4, pp. 369-377, 2006.

[16] X. Fu and X. Liu, "Controllability of non-densely defined neutral functional differential systems in abstract space," Chinese Annals of Mathematics Series B, vol. 28, no. 2, pp. 243-252, 2007.

[17] R. Sakthivel, Y. Ren, and N. I. Mahmudov, "Approximate controllability of second-order stochastic differential equations with impulsive effects," Modern Physics Letters B, vol. 24, no. 14, pp. 1559-1572, 2010.

[18] R. Sakthivel, J. J. Nieto, and N. I. Mahmudov, "Approximate controllability of nonlinear deterministic and stochastic systems with unbounded delay," Taiwanese Journal of Mathematics, vol. 14, no. 5, pp. 1777-1797, 2010.

[19] S. Ji, G. Li, and M. Wang, "Controllability of impulsive differential systems with nonlocal conditions," Applied Mathematics and Computation, vol. 217, no. 16, pp. 6981-6989, 2011.

[20] J. Klamka, A. Babiarz, and M. Niezabitowski, "Banach fixedpoint theorem in semilinear controllability problems-a survey," Bulletin of the Polish Academy of Sciences Technical Sciences, vol. 64, no. 1, pp. 21-35, 2016.

[21] A. Babiarz, J. Klamka, and M. Niezabitowski, "Schauders fixedpoint theorem in approximate controllability problems," International Journal of Applied Mathematics and Computer Science, vol. 26, no. 2, pp. 263-275, 2016.

[22] X. Zhang, C. Zhu, and C. Yuan, "Approximate controllability of fractional impulsive evolution systems involving nonlocal initial conditions," Advances in Difference Equations, vol. 2015, article 244, 2015.

[23] T. Guendouzi and S. Farahi, "Approximate controllability of Sobolev-type fractional functional stochastic integro-differential systems," Boletín de la Sociedad Matemática Mexicana, vol. 21, no. 2, pp. 289-308, 2015.

[24] I. Podlubny, Fractional Differential Equations: An Introduction to Fractional Derivatives, Fractional Differential Equations, to Methods of Their Solution and some of Their Applications, Elsevier Science, 1998.

[25] A. A. Kilbas, H. M. Srivastava, and J. J. Trujillo, Theory and Applications of Fractional Differential Equations, Elsevier, 2006. 
[26] V. Lakshmikantham, S. Leela, and J. V. Devi, Theory of Fractional Dynamic Systems, CSP, 2009.

[27] K. Diethelm, The Analysis of Fractional Differential Equations: An Application-Oriented Exposition Using Differential Operators of Caputo Type, Springer, 2010.

[28] C. A. Monje, Y. Q. Chen, B. M. Vinagre, D. Xue, and V. Feliu, Fractional-Order Systems and Controls: Fundamentals and Applications, Springer, London, UK, 2010.

[29] T. Kaczorek, Selected Problems of Fractional Systems Theory, Springer, Berlin, Germany, 2011.

[30] D. Baleanu, Fractional Calculus: Models and Numerical Methods, World Scientific Publishing, 2012.

[31] T. Kaczorek and L. Sajewski, The Realization Problem for Positive and Fractional Systems, vol. 1 of Studies in Systems, Decision and Control, Springer, 2014.

[32] R. Herrmann, Fractional Calculus: An Introduction for Physicists, World Scientific, 2014.

[33] O. Bachelier, P. Dabkowski, K. Gałkowski, and A. Kummert, "Fractional and $n \mathrm{D}$ systems: a continuous case," Multidimensional Systems and Signal Processing, vol. 23, no. 3, pp. 329-347, 2012.

[34] Z. Yan, "Controllability of fractional-order partial neutral functional integrodifferential inclusions with infinite delay," Journal of the Franklin Institute, vol. 348, no. 8, pp. 2156-2173, 2011.

[35] Z. Yan, "Approximate controllability of partial neutral functional differential systems of fractional order with statedependent delay," International Journal of Control, vol. 85, no. 8, pp. 1051-1062, 2012.

[36] A. Debbouche and D. F. Torres, "Approximate controllability of fractional delay dynamic inclusions with nonlocal control conditions," Applied Mathematics and Computation, vol. 243, pp. 161-175, 2014.

[37] Y. Zhou, V. Vijayakumar, and R. Murugesu, "Controllability for fractional evolution inclusions without compactness," Evolution Equations and Control Theory, vol. 4, no. 4, pp. 507-524, 2015.

[38] Y. Zhou, Fractional Evolution Equations and Inclusions: Analysis and Control, Academic Press, 2016.

[39] Z. Yan, "Existence results for fractional functional integrodifferential equations with nonlocal conditions in Banach spaces," Annales Polonici Mathematici, vol. 97, no. 3, pp. 285-299, 2010.

[40] Y. Zhou and F. Jiao, "Existence of mild solutions for fractional neutral evolution equations," Computers \& Mathematics with Applications, vol. 59, no. 3, pp. 1063-1077, 2010.

[41] S. Liang and R. Mei, "Existence of mild solutions for fractional impulsive neutral evolution equations with nonlocal conditions," Advances in Difference Equations, vol. 2014, article 101, 2014.

[42] A. Debbouche and J. J. Nieto, "Sobolev type fractional abstract evolution equations with nonlocal conditions and optimal multi-controls," Applied Mathematics and Computation, vol. 245, pp. 74-85, 2014.

[43] A. Debbouche and D. F. Torres, "Sobolev type fractional dynamic equations and optimal multi-integral controls with fractional nonlocal conditions," Fractional Calculus and Applied Analysis, vol. 18, no. 1, pp. 95-121, 2015.

[44] Z. Liu and X. Li, "On the controllability of impulsive fractional evolution inclusions in Banach spaces," Journal of Optimization Theory and Applications, vol. 156, no. 1, pp. 167-182, 2013.

[45] X. Zhang, X. Huang, and Z. Liu, "The existence and uniqueness of mild solutions for impulsive fractional equations with nonlocal conditions and infinite delay," Nonlinear Analysis: Hybrid Systems, vol. 4, no. 4, pp. 775-781, 2010.
[46] J. Wang and Y. Zhou, "Complete controllability of fractional evolution systems," Communications in Nonlinear Science and Numerical Simulation, vol. 17, no. 11, pp. 4346-4355, 2012.

[47] X.-F. Zhou, J. Wei, and L.-G. Hu, "Controllability of a fractional linear time-invariant neutral dynamical system," Applied Mathematics Letters, vol. 26, no. 4, pp. 418-424, 2013.

[48] R. P. Agarwal, B. de Andrade, and G. Siracusa, "On fractional integro-differential equations with state-dependent delay," Computers \& Mathematics with Applications, vol. 62, no. 3, pp. 1143-1149, 2011.

[49] B. de Andrade, S. Carvalho dos, and P. Jose, "Existence of solution for a fractional neutral integro-differential equation with unbounded delay," Electronic Journal of Differential Equations, vol. 2012, article 90, 2012.

[50] M. Fečkan, J. Wang, and Y. Zhou, "Controllability of fractional functional evolution equations of Sobolev type via characteristic solution operators," Journal of Optimization Theory and Applications, vol. 156, no. 1, pp. 79-95, 2013.

[51] K. Yosida, Functional Analysis, Springer, Berlin, Germany, 2013.

[52] P. M. Fitzpatrick and W. V. Petryshyn, "Fixed point theorems for multivalued noncompact acyclic mappings," Pacific Journal of Mathematics, vol. 54, no. 2, pp. 17-23, 1974.

[53] Y. Li, "Controllability of nonlinear neutral fractional impulsive differential inclusions in Banach space," Advances in Difference Equations, vol. 2014, article 234, 2014.

[54] R. F. Curtain and H. Zwart, An Introduction to InfiniteDimensional Linear Systems Theory, vol. 21, Springer Science \& Business Media, 1995.

[55] Z. Yan and X. Jia, "Approximate controllability of fractional impulsive partial neutral integrodifferential inclusions with infinite delay in Hilbert spaces," Advances in Difference Equations, vol. 2015, 2015.

[56] A. Lasota and Z. Opial, "An application of the Kakutani-Ky Fan theorem in the theory of ordinary differential equations," Bulletin de l'Académie Polonaise des Sciences, Série des Sciences Mathématiques, Astronomiques et Physiques, vol.13, pp. 781-786, 1965.

[57] R. P. Agarwal and D. O’Regan, "Leray-Schauder and Krasnoselskii results for multivalued maps defined on pseudo-open subsets of a Fréchet space," Applied Mathematics Letters, vol. 19, no. 12, pp. 1327-1334, 2006.

[58] B. C. Dhage, "Multivalued mapping and fixed point I," Nonlinear Functional Analysis and Applications, vol. 10, pp. 359-378, 2005.

[59] K. Jeet and D. Bahuguna, "Approximate controllability of nonlocal neutral fractional integro-differential equations with finite delay," Journal of Dynamical and Control Systems, vol. 22, no. 3, pp. 485-504, 2016.

[60] S. Kailasavalli, D. Baleanu, S. Suganya, and M. M. Arjunan, "Exact controllability of fractional neutral integro-differential systems with state-dependent delay in Banach spaces," Analele Stiintifice ale Universitatii Ovidius Constanta, Seria Matematica, vol. 24, no. 1, pp. 29-55, 2016.

[61] S. Suganya, M. M. Arjunan, and J. J. Trujillo, "Existence results for an impulsive fractional integro-differential equation with state-dependent delay," Applied Mathematics and Computation, vol. 266, pp. 54-69, 2015.

[62] Z. Yan, "Approximate controllability of fractional neutral integro-differential inclusions with state-dependent delay in Hilbert spaces," IMA Journal of Mathematical Control and Information, vol. 30, no. 4, pp. 443-462, 2013. 
[63] V. Vijayakumar, C. Ravichandran, and R. Murugesu, "Approximate controllability for a class of fractional neutral integrodifferential inclusions with state-dependent delay," Nonlinear Studies, vol. 20, no. 4, pp. 513-532, 2013.

[64] B. D. Andrade and J. P. C. Dos Santos, "Existence of solutions for a fractional neutral integrodifferential equation with unbounded delay," Electronic Journal of Differential Equations, vol. 2012, no. 90, 13 pages, 2012.

[65] V. Vijayakumar, A. Selvakumar, and R. Murugesu, "Controllability for a class of fractional neutral integro-differential equations with unbounded delay," Applied Mathematics and Computation, vol. 232, pp. 303-312, 2014.

[66] A. Pazy, Semigroups of Linear Operators and Applications to Partial Differential Equations, Springer, 1983.

[67] R. Ganesh, R. Sakthivel, Y. Ren, S. M. Anthoni, and N. I. Mahmudov, "Controllability of neutral fractional functional equations with impulses and infinite delay," Abstract and Applied Analysis, vol. 2013, Article ID 901625, 12 pages, 2013.

[68] R. Sakthivel, N. I. Mahmudov, and J. J. Nieto, "Controllability for a class of fractional-order neutral evolution control systems," Applied Mathematics and Computation, vol. 218, no. 20, pp. 10334-10340, 2012.

[69] S. Liu, A. Debbouche, and J. Wang, "On the iterative learning control for stochastic impulsive differential equations with randomly varying trial lengths," Journal of Computational and Applied Mathematics, vol. 312, no. 1, pp. 47-57, 2017.

[70] J. Wang and Y. Zhou, "A class of fractional evolution equations and optimal controls," Nonlinear Analysis: Real World Applications, vol. 12, no. 1, pp. 262-272, 2011.

[71] J. Wang, Y. Zhou, W. Wei, and H. Xu, "Nonlocal problems for fractional integrodifferential equations via fractional operators and optimal controls," Computers \& Mathematics with Applications, vol. 62, no. 3, pp. 1427-1441, 2011.

[72] J. Wang, Y. Zhou, and M. Medved', “On the solvability and optimal controls of fractional integrodifferential evolution systems with infinite delay," Journal of Optimization Theory and Applications, vol. 152, no. 1, pp. 31-50, 2012. 


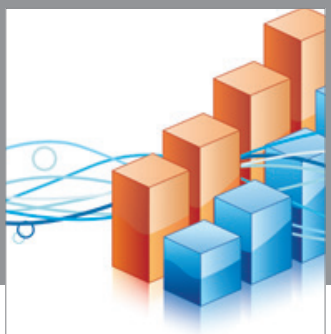

Advances in

Operations Research

vatem alat4

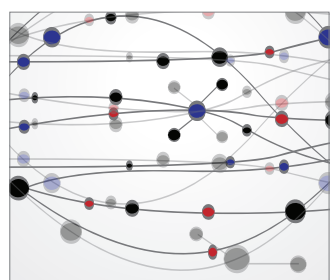

\section{The Scientific} World Journal
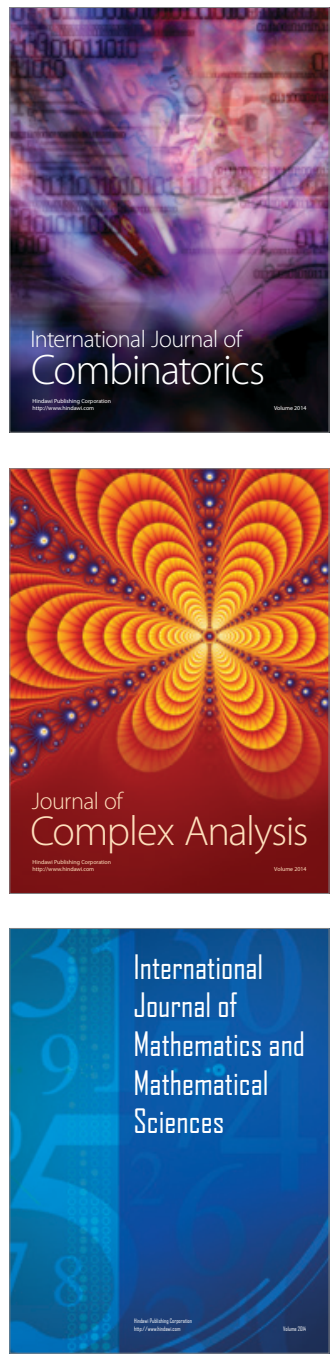
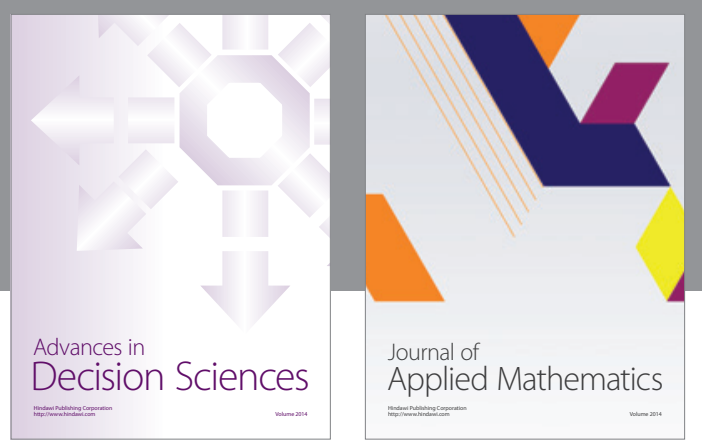

Algebra

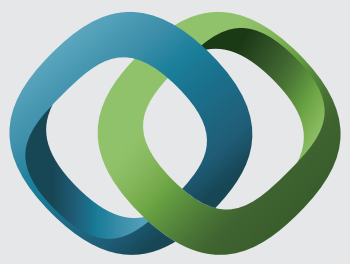

\section{Hindawi}

Submit your manuscripts at

https://www.hindawi.com
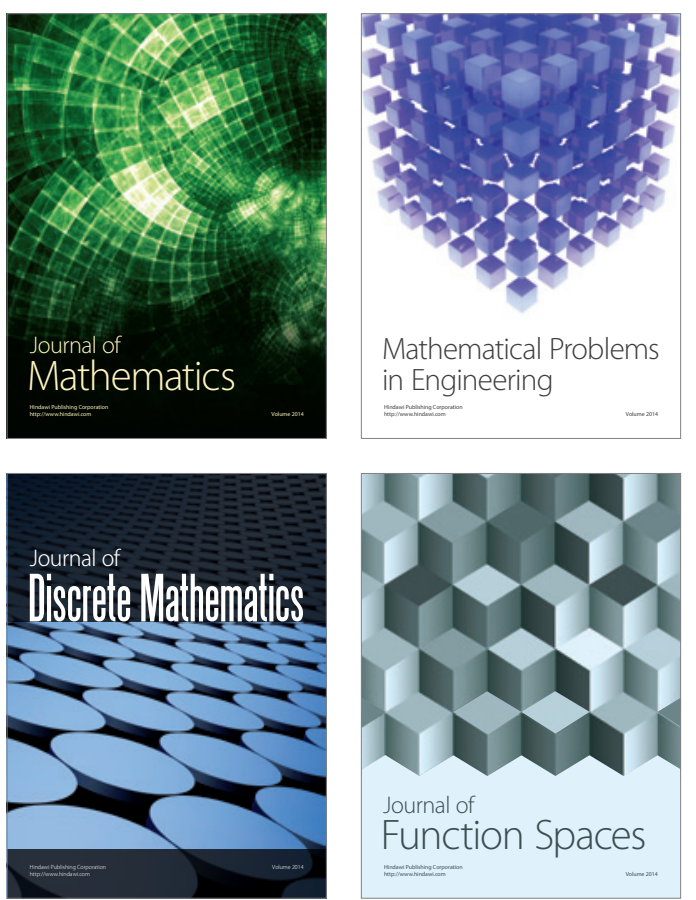

Mathematical Problems in Engineering
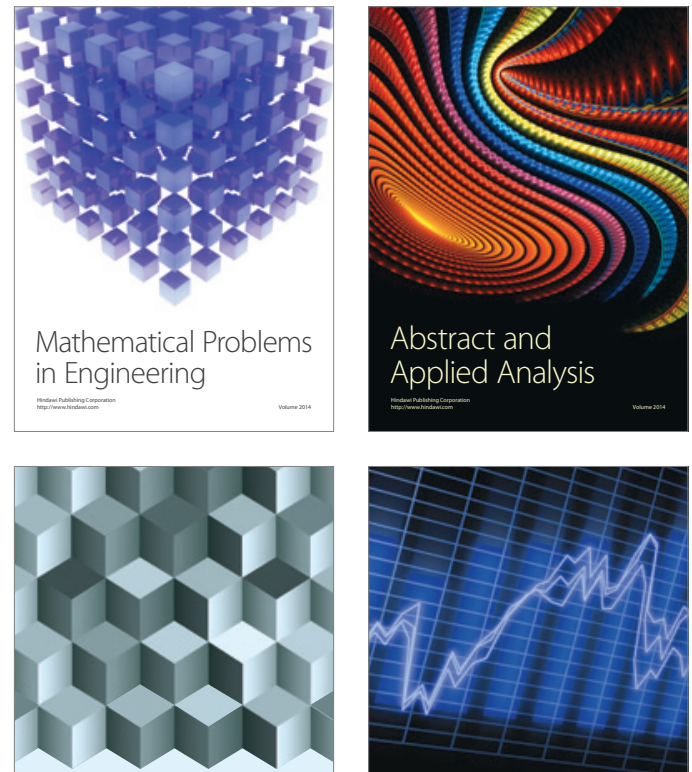

Journal of

Function Spaces

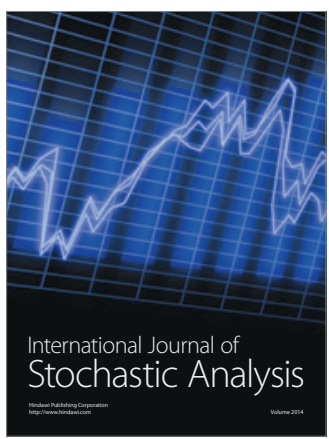

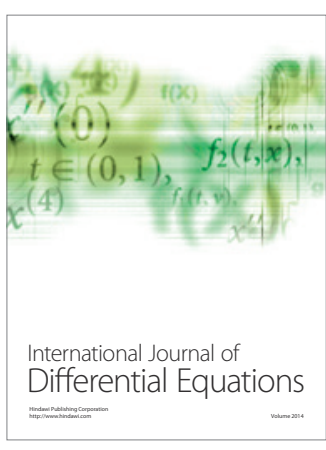
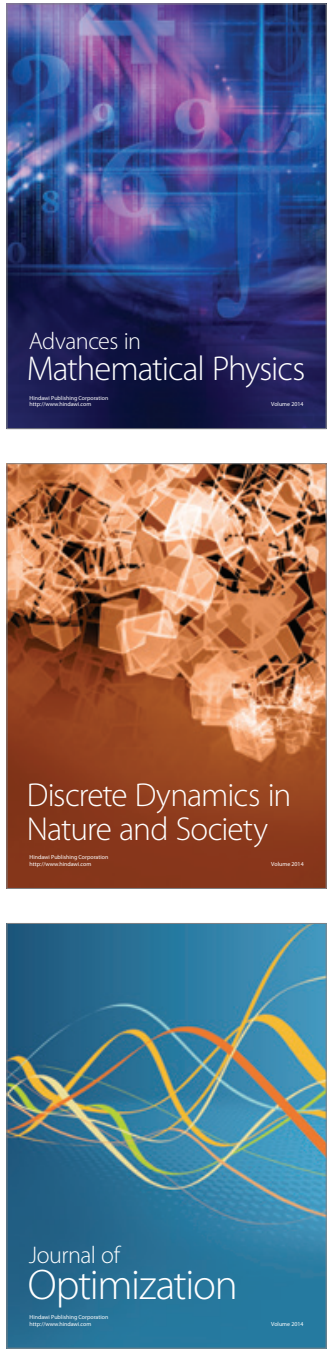